
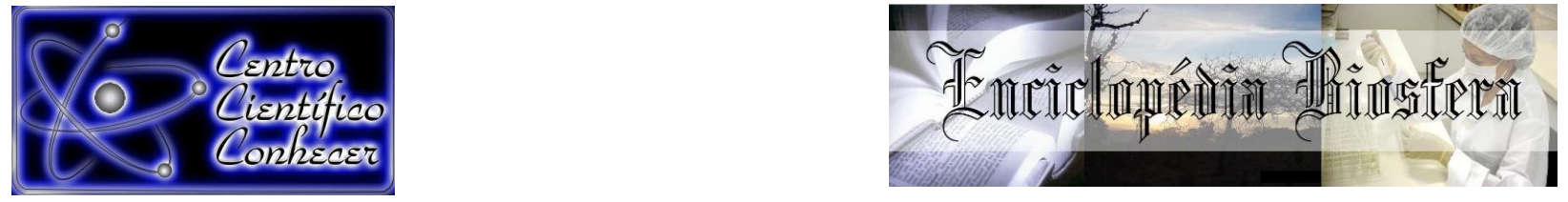

\title{
POTENCIAL FISIOLÓGICO DE SEMENTES DE GENÓTIPOS DE SOJA PRODUZIDOS EM DIFERENTES AMBIENTES DE CERRADO EM RORAIMA
}

Hananda Hellen da Silva Gomes ${ }^{1}$; Oscar José Smiderle ${ }^{2}$; Vicente Gianluppi ${ }^{3}$ Daniel Gianluppi ${ }^{3}$.

${ }^{1}$ Estudante de Agronomia - UFRR, Bolsista - PIC/PIBIT - CNPq, LAS da Embrapa Roraima (hananda_hellen@hotmail.com), Boa Vista - Roraima;

${ }^{2}$ Pesquisador A, Embrapa Roraima - CPAF-RR, Boa Vista - Roraima;

${ }^{3}$ Pesquisador B, Embrapa Roraima - CPAF-RR, Boa Vista - Roraima

Recebido em: 06/04/2018 - Aprovado em: 10/06/2018 - Publicado em: 20/06/2018 DOI: 10.18677/EnciBio_2018A68

\begin{abstract}
RESUMO
Vários fatores podem determinar a qualidade fisiológica das sementes, dentre eles pode-se ressaltar o genótipo ocasionado pela maturação fisiológica, definido pelo peso de massa seca, germinação e o vigor. Objetivou-se determinar o potencial fisiológico de sementes de genótipos de soja produzidas em três ambientes de cultivo no cerrado em Roraima. Os experimentos foram instalados em três ambientes no cerrado de Roraima e no Laboratório de Análise de Sementes (LAS) da Embrapa Roraima em 2017. Foram avaliadas sementes de soja de trinta e cinco genótipos produzidas em três ambientes (Campo experimental Água boa/Embrapa RR - AB, Fazenda Livramento - FL, Fazenda São Pedro - FSP) para avaliação de massa de 1000 sementes, produtividade, primeira contagem de germinação, teste de germinação, emergência de plântulas, velocidade de emergência em campo e condutividade elétrica. O delineamento utilizado no campo foi em blocos e no laboratório foi inteiramente casualizado formado pelo esquema fatorial $(3 \times 35)$. Os dados obtidos das variáveis mensuradas foram submetidos à análise de variância com aplicação do Teste de Scott-Knott para diferentes genótipos e Teste de Tukey para diferentes ambientes a $5 \%$ de probabilidade. Verificou-se que houve interação significativa a $5 \%$ de probabilidade entre ambientes e os genótipos analisados. Para as variáveis mensuradas, a MSoy 8372 IPRO produz sementes com maior vigor no ambiente Água Boa, a BRASBT13-0225 no ambiente Fazenda Livramento, e a Sambaiba 05 IPRO no ambiente Fazenda São Pedro. Nenhum dos 35 genótipos apresenta maior vigor nos três ambientes. O ambiente Fazenda Livramento produz mais sementes e com menor vigor.
\end{abstract}

PALAVRAS-CHAVE: Glycine max (L.). Vigor. Qualidade de sementes.

\section{PHYSIOLOGICAL POTENTIAL OF SEEDS OF 35 SOYBEAN GENOTYPES PRODUCED IN DIFFERENT CLIMATE ENVIRONMENTS IN RORAIMA}

\begin{abstract}
Several factors can determine the physiological quality of the seeds, among them we can highlight the genotype caused by the physiological maturation, defined by the weight of dry mass, germination and vigor. The objective was to determine the physiological potential of soybean genotype seeds produced in three cultivation
\end{abstract}


environments in the cerrado of Roraima. The experiments were carried out in three environments in the cerrado of Roraima and in the Laboratory of Seed Analysis (LAS) of Embrapa Roraima in 2017. Soybean seeds of thirty - five genotypes produced in three environments were evaluated (Água boa experimental field / Embrapa RR - AB, Fazenda Livramento - FL, Fazenda São Pedro - FSP) for evaluation of mass of 1000 seeds, productivity, first germination count, germination test, emergence of field seedlings, field emergence velocity and electrical conductivity. The design used in the field was in blocks and in the laboratory was completely randomized formed by the factorial scheme $(3 \times 35)$. The data obtained from the measured variables were submitted to analysis of variance with application of the Scott-Knott test for different genotypes and Tukey's test for different environments at $5 \%$ probability. It was verified that there was significant interaction at $5 \%$ of probability between environments and the analyzed genotypes. For the variables measured, M. Soy 8372 IPRO produces seeds with greater vigor in the Água Boa environment, while BRASBT13-0225 in Farm Livramento environment, and Sambaiba 05 IPRO in the environment Farm Saint Peter. None of the 35 genotypes present greater vigor in the three environments. The Farm Livramento environment produces more seeds and less vigor.

KEYWORDS: Glycine max (L.). Vigor. Seed quality.

\section{INTRODUÇÃO}

A soja pertencente a família botânica das Fabáceas, se caracteriza como a principal oleaginosa produzida no Brasil, devido ao ambiente de produção que vem crescendo ao longo dos últimos 20 anos e tornando o país como o segundo maior produtor de soja do mundo (CONAB, 2017).

Estima-se que a safra brasileira 2017/2018 tenha área plantada de 35,3 milhões de hectares com produtividade média de $3075 \mathrm{~kg} \mathrm{ha}^{-1}$ e produção de 108,6 milhões de toneladas de grãos. No estado de Roraima a safra 2017/2018 terá área plantada próxima de 30 mil hectares com produtividade de $3000 \mathrm{~kg} \mathrm{ha}^{-1}$ e produção de 92,3 mil toneladas de grãos (CONAB, 2017).

O cerrado de Roraima, segundo a classificação de Köppen, apresenta clima tipo Aw tropical chuvoso, com precipitação média anual de aproximadamente 1800 $\mathrm{mm}$ e concentração de mais de $80 \%$ das chuvas entre os meses de abril e setembro e estiagem de até 120 dias (SMIDERLE, 2018), favorecendo o plantio de soja no estado (SILVA et al., 2016). Altas produtividades só serão obtidas quando as condições de semeaduras forem adequadas com o uso de sementes de qualidade, assim como condições ambientais apropriadas para todas as fases vegetativas da planta.

Para que se tenha qualidade nas sementes de soja, é necessário: qualidade fisiológica apresentando sementes com alto vigor e germinação que reflete em satisfatória emergência em campo; qualidade genética, sendo genótipos puros, sem misturas parentais representando a cultivar que se deseja plantar; ideal que se tenha qualidade sanitária, livre de patógenos ou plantas invasoras; qualidade física, sementes livres de contaminantes, fragmentos indesejáveis, torrões e outras impurezas (FRANÇA NETO et al., 2016).

Vários fatores podem determinar a qualidade fisiológica das sementes, dentre as quais pode-se ressaltar o genótipo ocasionado pela maturação fisiológica, definido pelo peso de massa seca, germinação e o vigor. Com a alta apresentação desses fatores, pode-se definir o desempenho das sementes em condições armazenadas e em campo. Dessa maneira, só pode ser comprovada a eficiência da 
avaliação laboratorial a partir do momento em que as plantas forem semeadas no campo.

Desse modo, o teste de vigor aliado com a germinação é importante, pois o vigor das sementes determina a capacidade de emergência das plantas, possibilitando respostas em diferentes ambientes. Assim, são ideais que se realizem testes de germinação e vigor antes da semeadura para o estabelecimento da cultura no campo, determinar assim os genótipos fisiologicamente superiores.

Desta forma, quando são utilizados novos genótipos no programa de melhoramento, é ideal que sejam feitos testes de germinação e vigor que são critérios para atingir o potencial fisiológico, distinguindo lotes com maior ou menor eficácia de campo, que podem produzir sementes vigorosas ou não.

Neste contexto, objetivou-se determinar o potencial fisiológico de sementes de genótipos de soja produzidas em três ambientes de cultivo no cerrado em Roraima.

\section{MATERIAL E MÉTODOS}

Os experimentos foram instalados em 2017 em três ambientes no cerrado de Roraima e no Laboratório de Análise de Sementes (LAS) da Embrapa Roraima. Foram avaliadas sementes de soja de 35 lotes, representados pelos genótipos: BRS 7380; BRS 7680; BRS 8781; BRS 9280; MSoy 7739 IPRO; MSoy 8372 IPRO; BRS 9180 IPRO; BRS 9383 IPRO; Sambaiba 05 IPRO; BRASRR12-10657; BRASRR1214296; BRASRR12-13666; BRRY45-16300; BRRY45-16283; BRASRR12-13039; BRRY45-16301; BRASBT13-0016; BRASBT13-0758; BRASBT13-0691; BRASBT130590; BRASBT13-0621; BRASBT13-0872; BRASBT13-0715; BRASBT13-0762; BRASBT13-0834; $\quad$ BRASBT13-0843; BRASBT13-0054; $\quad$ BRASBT13-0176; BRASBT13-0200; BRASBT13-0528; BRASBT13-0553; BRASBT13-0225; BRASBT13-0646; BRASBT13-0698; BRASBT13-064. Produzidas em três ambientes de cultivo no cerrado de Roraima: Campo experimental Água Boa - Embrapa RR (AB) no município de Boa Vista - RR, com coordenadas geográficas de referência registradas a $02^{\circ} 40^{\prime} 04.9^{\prime \prime}$ de latitude, $60^{\circ} 50^{\prime} 33.4^{\prime \prime}$ de longitude e $90 \mathrm{~m}$ de altitude; Fazenda Livramento - AC (a 02 49' 39.1" de latitude N, W60 34' 50.7'" a 84 msnm), Fazenda São Pedro (FSP) a 02 ${ }^{\circ} 37^{\prime}$ '56.7" de latitude N, W60 53' 16.7' a 88 msnm.

Foram realizadas nos três ambientes, adubação de plantio com aplicação de $450 \mathrm{~kg} \mathrm{ha}^{-1}$ de N-P-K (04-24-12 + micros). A adubação de cobertura foi realizada aos 20 dias após a emergência das plântulas, constando de $200 \mathrm{~kg} \mathrm{ha}^{-1}$ de cloreto de potássio. Os demais tratos culturais foram realizados conforme o sistema de cultivo da soja para o Cerrado de Roraima (SMIDERLE et al., 2016).

O delineamento utilizado no campo foi em blocos e no laboratório foi inteiramente casualizado sendo formado pelo esquema fatorial (3x35), 3 ambientes de produção de sementes e 35 genótipos de soja com quatro repetições. As parcelas experimentais foram compostas por quatro linhas com $5 \mathrm{~m}$ de comprimento, espaçadas por $0,5 \mathrm{~m}\left(10,0 \mathrm{~m}^{2}\right)$. Após a colheita e trilha mecânica das plantas obtidas da área útil das parcelas $\left(5 \mathrm{~m}^{2}\right)$, as sementes foram pesadas e em seguida foi calculada a produtividade de sementes em $\mathrm{kg} \mathrm{ha}^{-1}$, com correção para $13 \%$.

Foi retirada uma amostra de 0,5 kg de sementes de cada genótipo, após o beneficiamento, aonde foram submetidas às avaliações para determinação da qualidade fisiológica das sementes sendo: Massa de 1000 sementes, grau de umidade, germinação, vigor, condutividade elétrica, emergência de plântulas e velocidade de emergência de plântulas em canteiros de areia. 
Para avaliação da massa de 1000 sementes, estas foram separadas com o auxílio de um tabuleiro contador, em oito repetições contendo 100 sementes de cada genótipo, que foram pesadas em balança de precisão de 0,001 $\mathrm{g}$, e os valores expressos em gramas após correção do teor de água para $13 \%$.

O teor de água foi determinado pelo método da estufa a $105^{\circ} \mathrm{C} \pm 5$, durante 24 horas utilizando duas amostras de $10 \mathrm{~g}$ de sementes, para cada genótipo e os dados expressos em porcentagem média (BRASIL, 2009).

Teste de germinação, realizado com quatro repetições de 50 sementes de cada material, as quais foram colocadas em papel germitest umedecido com água destilada 2,5 vezes o peso do papel seco em gramas, no qual foram colocadas para germinar em duas folhas dobradas cuidadosamente para a confecção de rolos com as sementes distribuídas em forma linear. Em seguida, colocadas na câmara de germinação com temperatura constante de $25^{\circ} \mathrm{C}$. A avaliação da germinação foi realizada no oitavo dia após a instalação, contagem das plântulas normais conforme especificado nas Regras para Análise de Sementes e os resultados expressos em porcentagem (BRASIL, 2009).

A primeira contagem da germinação, realizada em conjunto com o teste de germinação, utilizando a mesma metodologia, consistiu na contagem de plântulas normais realizada no quinto dia após a instalação do teste, considerando as que apresentaram as partes morfológicas completas e sem lesões (BRASIL, 2009).

$\mathrm{Na}$ avaliação da condutividade elétrica, foi utilizado quatro repetições contendo 50 sementes de cada genótipo, colocadas em copos plásticos de $180 \mathrm{~mL}$ e pesadas em balança de precisão de $0,001 \mathrm{~g}$ e em seguida, colocadas para embeber com $75 \mathrm{~mL}$ de água deionizada durante 24 horas em temperatura de $25^{\circ} \mathrm{C}$. Após as 24 horas foi realizada a leitura da condutividade em condutivímetro digital, em seguida retirada a água e pesadas as sementes úmidas. Os resultados foram expressos em $\mu S . \mathrm{cm}^{-1} \mathrm{~g}^{-1}$ de semente depois de realizada a divisão pela massa inicial das 50 sementes (CE), bem como pela massa final (CE24).

Emergência das plântulas em substrato de areia, foi realizada com duas repetições de 50 sementes de cada genótipo semeadas em canteiro de areia, em sulcos longitudinais de um metro de comprimento e espaçamento de 0,10 metros entre si, com o auxílio de sulcador plástico de pvc. O teste foi realizado em casa de vegetação da Embrapa Roraima e a umidade mantida com irrigação automatizada em quatro turnos diários de 5 minutos cada. As contagens foram realizadas diariamente após emergidos completamente os cotilédones. No final do décimo quarto dia após a semeadura, não sendo mais observado acréscimo da emergência de novas plântulas, foi finalizado a contagem e obtida a percentagem de plântulas normais. Os resultados foram expressos em porcentagem (BRASIL, 2009).

Velocidade de emergência de plântulas foi obtida junto com a emergência de plântulas em canteiros de areia, em que foram realizadas diariamente anotações da contagem do número de plântulas emergidas. Assim, com o número de plântulas emergidas, referente as leituras realizadas em casa de vegetação, calculou-se a velocidade de emergência (VE) de acordo com a formula de Maguire (1962).

Os dados foram submetidos à análise de variância (teste $F$ a $5 \%$ de probabilidade $(p \leq 0,05))$, de modo a avaliarem-se os efeitos dos tratamentos em cada variável. $\mathrm{O}$ agrupamento das médias dos genótipos realizado pelo teste de ScottKnott $(p \leq 0,05)$ e a comparação de médias entre os ambientes com teste de Tukey a $5 \%$ de probabilidade. Foi utilizado o software SISVAR 5.4. 


\section{RESULTADOS E DISCUSSÃO}

De acordo com os resultados obtidos, observou-se que houve interação significativa a $5 \%$ de probabilidade entre os ambientes e trinta e cinco genótipos analisados, para as variáveis: massa de 1000 sementes e produtividade de grãos (Tabela 1).

TABELA 1 - Resultado da análise de variância para massa de 1000 sementes (M1000S), produtividade de grãos (PROD), germinação $(\mathrm{G})$, primeira contagem de germinação (PCG), emergência em campo $(E C)$, velocidade de emergência de plântulas em campo (VE) e condutividade elétrica (CE/CE24) obtidas em sementes de 35 genótipos produzidas em três ambientes do cerrado de Roraima

\begin{tabular}{cccccccccc}
\hline FV & GL & M1000S & PROD & G & PCG & EC & VE & CE & CE24 \\
\hline AMB (A) & 2 & $82,8^{*}$ & $56672186,6^{*}$ & $40908,2^{*}$ & $11613,1^{*}$ & $13353,1^{*}$ & $1908,9^{*}$ & $49513,3^{*}$ & $9234,3^{*}$ \\
GENÓTIPO & 34 & $32,7^{*}$ & $2874503,1^{*}$ & $2958,5^{*}$ & $666,8^{*}$ & $4079,9^{*}$ & $217,0^{*}$ & $8063,4^{*}$ & $1274,3^{*}$ \\
(G) & 68 & $8,1^{*}$ & $1421621,4^{*}$ & $1738,6^{*}$ & $393,6^{*}$ & $1786,6^{*}$ & $97,6^{*}$ & $4449,6^{*}$ & $719,2^{*}$ \\
A x G & 68 & 2,57 & 837191,42 & 30,10 & 6,92 & 1,21 & 0,01 & 92,28 & 16,34 \\
Erro & 315 & 2,57 & 29,37 & 12,86 & 13,07 & 2,94 & 0,91 & 5,22 & 5,42 \\
\hline CV \% & & 2,57 &
\end{tabular}

* Significativo a $1 \%$ de probabilidade pelo teste $\mathrm{F}$.

O baixo coeficiente de variação $(2,57 \%)$, verificado na Tabela 1 destaca o ambiente FL com variação de 230,67 a 153,04 g tendo destaque para os genótipos BRS 8781, BRASRR12-13039 e BRASBT13-0691 que apresentaram maior massa em relação aos demais. Embora o genótipo BRS 8781 tenha se destacado por ter apresentado uma das maiores massas de 1000 sementes, a produtividade não foi satisfatória, tendo diferença significativa em relação às demais com maiores produtividades. Pode-se observar que no ambiente FL obteve-se maior produção de sementes em relação aos demais (Tabela 2).

Em relação ao ambiente FSP, destacaram-se os genótipos BRS 8781 e BRASBT13-0590 como os de maior massa de 1000 sementes produzidas no ambiente. Embora a produtividade do BRS 8781 tenha se destacado como uma das melhores, o mesmo não ocorreu com a BRASBT13-0590 que apresentou produtividade estatisticamente inferior aos demais (Tabela 2).

TABELA 2. Valores médios de massa de mil sementes (M1000s) e produtividade de grãos (PROD) obtidas de sementes de trinta e cinco genótipos produzidas em três ambientes do cerrado de Roraima

\begin{tabular}{|c|c|c|c|c|c|c|c|c|c|c|}
\hline \multirow{2}{*}{ GENÓTIPOS } & \multicolumn{5}{|c|}{ M1000S (g) } & \multicolumn{5}{|c|}{ PROD (kg ha-1) } \\
\hline & \multicolumn{2}{|c|}{ AB } & $\mathrm{FL}$ & & FSP & \multicolumn{2}{|c|}{$A B$} & \multirow{2}{*}{\multicolumn{2}{|c|}{ FL }} & FSP \\
\hline BRRY45-16301 & 164,14 & $f A B^{*}$ & 171,35 & $\mathrm{gA}$ & $157,45 \mathrm{hB}$ & 3025 & $\mathrm{aB}$ & & & 2457 bB \\
\hline BRASBT13-0016 & 181,61 & $\mathrm{eB}$ & 187,83 & $\mathrm{eB}$ & $198,02 \mathrm{dA}$ & 2760 & $\mathrm{aB}$ & 4857 & $\mathrm{aA}$ & 5122 aA \\
\hline BRASBT13-0698 & 165,72 & $f B$ & 176,41 & $\mathrm{fA}$ & $163,3 \mathrm{gB}$ & 2775 & $a B$ & 4547 & $\mathrm{aA}$ & $2822 b B$ \\
\hline BRASBT13-0553 & 178,66 & $e B$ & 194,87 & $d A$ & $166,97 \mathrm{gC}$ & 1950 & $\mathrm{bB}$ & 4497 & $\mathrm{aA}$ & $1960 \mathrm{bB}$ \\
\hline BRS 9280 & 179,88 & $\mathrm{eC}$ & 204,42 & $c A$ & $193,72 \mathrm{~dB}$ & 3305 & $\mathrm{aA}$ & 4487 & $\mathrm{aA}$ & 3802 aA \\
\hline BRASRR12-13039 & 187,33 & $d B$ & 230,67 & $\mathrm{aA}$ & $181,2 \mathrm{fB}$ & 1875 & $\mathrm{bB}$ & 4450 & $\mathrm{aA}$ & $2830 \mathrm{bB}$ \\
\hline BRRY45-16283 & 172,87 & eA & 154,56 & iB & $153,03 \mathrm{hB}$ & 3225 & $\mathrm{aA}$ & 4425 & $\mathrm{aA}$ & 3342 aA \\
\hline BRASBT13-0641 & 178,37 & $\mathrm{eA}$ & 180,26 & $\mathrm{fA}$ & 183,85 fA & 1920 & $\mathrm{bB}$ & 4415 & $\mathrm{aA}$ & $2730 \mathrm{bB}$ \\
\hline BRASRR12-14296 & 167,82 & fC & 217,86 & bA & $188,18 \mathrm{eB}$ & 1955 & $\mathrm{bB}$ & 4397 & $\mathrm{aA}$ & $1920 \mathrm{bB}$ \\
\hline BRASBT13-0691 & 207,69 & $c B$ & 227,45 & $a A$ & $179,27 \mathrm{fC}$ & 2835 & $a B$ & 4392 & $\mathrm{aA}$ & $2040 \mathrm{bB}$ \\
\hline BRASBT13-0621 & 192,42 & $d A$ & 190,34 & eA & 191,48 eA & 3060 & $\mathrm{aA}$ & 4380 & $\mathrm{aA}$ & 3162 aA \\
\hline BRASBT13-0834 & 166,89 & $f B$ & 188,39 & eA & 180,86 fA & 2760 & $a B$ & 4340 & $\mathrm{aA}$ & 2327 bB \\
\hline BRS 7680 & 226,1 & $\mathrm{aA}$ & 197,73 & $d B$ & $201,35 \quad c B$ & 1750 & $b B$ & 4147 & $\mathrm{aA}$ & $2130 \mathrm{bB}$ \\
\hline BRASBT13-0176 & 178,19 & $\mathrm{eC}$ & 186,57 & $d B$ & $199,23 d A$ & 2625 & $a A B$ & 4055 & $\mathrm{aA}$ & $1755 \mathrm{bB}$ \\
\hline BRASBT13-0200 & 179,13 & $\mathrm{eB}$ & 195,8 & $\mathrm{eA}$ & 191,29 eA & 3225 & $\mathrm{aA}$ & 3975 & $\mathrm{aA}$ & 3277 aA \\
\hline ruYaginǵn & & & & & & & & 236 & & 2018 \\
\hline
\end{tabular}




\begin{tabular}{|c|c|c|c|c|c|c|c|c|c|c|c|c|}
\hline BRASBT13-0872 & 179,97 & $e B$ & 205,04 & cA & 204,87 & cA & 3090 & $\mathrm{aA}$ & 3832 & bA & 3760 & $\mathrm{aA}$ \\
\hline BRS 9180 IPRO & 164,87 & $f B$ & 202,82 & $c A$ & 158,04 & hB & 2720 & $a A$ & 3802 & bA & 3500 & $\mathrm{aA}$ \\
\hline Sambaiba 05 IPRO & 154,53 & $\mathrm{hC}$ & 183,16 & $e B$ & 215,73 & bA & 3595 & $\mathrm{aA}$ & 3770 & bA & 4460 & $\mathrm{aA}$ \\
\hline BRASBT13-0646 & 162,97 & $\mathrm{fC}$ & 191,31 & $e B$ & 203,65 & $c A$ & 2850 & $\mathrm{aA}$ & 3757 & bA & 3015 & $\mathrm{aA}$ \\
\hline BRASBT13-0762 & 162,15 & gB & 193,79 & $d A$ & 186,12 & eA & 2930 & $a A$ & 3690 & bA & 2322 & bA \\
\hline BRS 8781 & 192,39 & $\mathrm{~dB}$ & 222,65 & $a A$ & 225,01 & $\mathrm{aA}$ & 2380 & bA & 3660 & bA & 3387 & $\mathrm{aA}$ \\
\hline BRASBT13-0054 & 156,78 & gB & 174,25 & $\mathrm{fA}$ & 174,29 & fA & 3130 & $a A$ & 3575 & bA & 2747 & bA \\
\hline BRASBT13-0590 & 211,37 & $\mathrm{bB}$ & 215,29 & $\mathrm{bB}$ & 231,8 & $\mathrm{aA}$ & 1195 & $\mathrm{bB}$ & 3520 & bA & 2307 & bAB \\
\hline BRASRR12-13666 & 190,02 & $d B$ & 203,31 & $c A$ & 204,93 & $c A$ & 2195 & bA & 3450 & bA & 2392 & bA \\
\hline BRASRR12-10657 & 144,71 & iB & 167,65 & $g A$ & 137,46 & iB & 3585 & $\mathrm{aA}$ & 3415 & bA & 3125 & $\mathrm{aA}$ \\
\hline BRASBT13-0843 & 166,04 & $f B$ & 153,04 & $\mathrm{iC}$ & 202,69 & $c A$ & 2830 & $a A$ & 3400 & bA & 3220 & $\mathrm{aA}$ \\
\hline BRASBT13-0528 & 181,37 & $\mathrm{eC}$ & 198,84 & $d A$ & 190,08 & $\mathrm{eB}$ & 2725 & $a A$ & 3352 & bA & 3180 & $\mathrm{aA}$ \\
\hline BRASBT13-0225 & 194,32 & $d A$ & 185,52 & $e B$ & 186,15 & $e B$ & 1910 & bA & 3327 & bA & 3430 & $\mathrm{aA}$ \\
\hline BRASBT13-0715 & 195,54 & $\mathrm{eA}$ & 189,9 & eA & 193,94 & $d A$ & 1910 & $\mathrm{bAB}$ & 3322 & bA & 1720 & bB \\
\hline MSoy 8372 IPRO & 182,48 & $e B$ & 215,84 & bA & 210,72 & bA & 2775 & $\mathrm{aA}$ & 3315 & bA & 3747 & $\mathrm{aA}$ \\
\hline BRS 9383 IPRO & 149,59 & $\mathrm{hC}$ & 201,79 & $c A$ & 161,84 & gB & 3880 & $a A$ & 3242 & bA & 3855 & $\mathrm{aA}$ \\
\hline BRS 7380 & 205,3 & bA & 206,73 & $c A$ & 173,61 & $\mathrm{fB}$ & 1685 & bA & 3130 & bA & 1837 & bA \\
\hline MSoy 7739 IPRO & 200,33 & $c B$ & 217,41 & $\mathrm{bA}$ & 214,05 & bA & 2280 & bA & 3035 & bA & 2140 & bA \\
\hline BRRY45-16300 & 136,07 & jB & 161,25 & $\mathrm{hA}$ & 164,45 & $g A$ & 2605 & $a A$ & 2750 & bA & 3797 & $\mathrm{aA}$ \\
\hline BRASBT13-0758 & 197,79 & eB & 196,18 & $\mathrm{~dB}$ & 209,35 & $\mathrm{bA}$ & 2130 & bA & 2382 & bA & 1970 & bA \\
\hline CY\% & 2,5 & & 2,57 & & 2,57 & & & & & & & \\
\hline
\end{tabular}

"Médias seguidas por letras distintas, minúsculas na coluna e maiúsculas na linha, diferem entre si pelos testes de Scott-Knott e Tukey a $5 \%$ de probabilidade, respectivamente.

Tais resultados demonstraram que embora o genótipo BRS 8781 tenha apresentado maior massa de 1000 sementes em dois ambientes, não se caracteriza pela qualidade fisiológica das sementes produzidas. Possivelmente, esse fator pode ser explicado por condições adequadas durante a fase de enchimento dos grãos (Tabela 2).

Com isso, pode-se entender que no ambiente $A B$, o período de enchimento dos grãos, não teve condições adequadas para os genótipos analisados, apresentando variação média de 226,10 a $136,07 \mathrm{~g}$ sendo o ambiente que apresentou menores valores em comparação aos demais ambientes analisados. Embora a massa de 1000 sementes tenha apresentado baixos valores, a produtividade não foi afetada em relação aos demais ambientes.

De acordo com a análise de variância dos três ambientes de produção de sementes (Tabela 2), destacaram-se as linhagens BRASBT13-0621, BRASBT130715 e BRASBT13-0641 que não se diferenciaram estatisticamente nos três ambientes. Porém, apesar dos genótipos não se diferenciarem significativamente, na massa de 1000 sementes não tiveram resultados satisfatórios em comparação aos demais genótipos. Em contrapartida, pode-se destacar o genótipo BRASBT13-0621 como produtivo nos três ambientes, e a BRASBT13-0715 como pouco produtiva. Não sendo a produtividade relacionada diretamente com a massa de 1000 sementes.

As médias de produtividade de grãos obtidas apresentaram diferença significativa entre os genótipos e os três ambientes, verificando-se maior produtividade nos genótipos BRS 9280, BRRY45-16283, BRASBT13-0016, BRASBT13-0621, BRASBT13-0200 nos três ambientes.

$\mathrm{Na}$ Tabela 3, os resultados obtidos para primeira contagem de germinação e germinação apresentaram diferença significativa, pela análise de variância, tanto entre os diferentes genótipos quanto nos diferentes ambientes, podendo assim determinar o potencial fisiológico dos genótipos nos ambientes de produção.

Com isso, pode-se destacar 0 ambiente $A B$ que apresentou os maiores valores de ambas as avaliações, evidenciando os genótipos MSoy 8372 IPRO, ENCICLOPÉDIA BIOSFERA, Centro Científico Conhecer - Goiânia, v.15 n.27; p. 237 
BRASBT13-0762, BRASBT13-0843 e BRASBT13-0054 que apresentaram vigor maior que $42 \%$ e germinação acima de $83 \%$ (Tabela 3 ).

No ambiente $\mathrm{FL}$, os resultados se diferenciaram em relação aos demais ambientes por apresentarem valores baixos de primeira contagem de germinação com 33\% de vigor pelo genótipo BRASBT13-0225. Enquanto os melhores valores de Germinação foram obtidos pelos genótipos BRASBT13-0225 e MSoy 8372 IPRO com 69 e 66\% respectivamente. Embora o ambiente tenha se destacado com maior produção de sementes, pode-se observar que sua produção foi de sementes com baixo vigor.

Embora o teste de germinação na tecnologia de sementes se dê pela emergência e o desenvolvimento das estruturas essenciais do embrião, a primeira contagem de germinação indica a velocidade das atividades fisiológicas da semente, indicando que o maior número de plantas germinadas no quinto dia de avaliação, será o lote de sementes de maior vigor.

TABELA 3. Médias de primeira contagem de germinação (PCG, em \%) e germinação ( $G$, em \%), de sementes de 35 genótipos produzidas em três ambientes diferentes no cerrado de Roraima

\begin{tabular}{|c|c|c|c|c|c|c|c|c|c|c|c|}
\hline \multirow{3}{*}{$\begin{array}{l}\text { GENÓTIPOS } \\
\text { BRASBT13-0843 }\end{array}$} & \multicolumn{5}{|c|}{ PCG } & \multicolumn{6}{|c|}{$\mathbf{G}$} \\
\hline & \multicolumn{2}{|c|}{ AB } & \multicolumn{2}{|c|}{ FL } & FSP & \multicolumn{2}{|c|}{ AB } & \multicolumn{2}{|c|}{ FL } & \multicolumn{2}{|c|}{ FSP } \\
\hline & 46 & $a A^{*}$ & 28 & $\mathrm{bC}$ & $35 \mathrm{bB}$ & 91 & $\mathrm{aA}$ & 59 & $\mathrm{bC}$ & 73 & $\mathrm{bB}$ \\
\hline MSoy 8372 IPRO & 45 & $a A$ & 23 & $\mathrm{cB}$ & $21 \mathrm{eB}$ & 92 & $\mathrm{aA}$ & 66 & $\mathrm{aB}$ & 53 & $\mathrm{dC}$ \\
\hline BRASBT13-0762 & 45 & $\mathrm{aA}$ & 25 & $\mathrm{cC}$ & $34 \mathrm{bB}$ & 90 & $\mathrm{aA}$ & 51 & $\mathrm{cC}$ & 81 & $\mathrm{aB}$ \\
\hline BRASBT13-0054 & 43 & $\mathrm{aA}$ & 17 & $\mathrm{eC}$ & $31 \mathrm{cB}$ & 86 & $\mathrm{aA}$ & 35 & $\mathrm{eC}$ & 66 & $\mathrm{cB}$ \\
\hline BRS 8781 & 42 & $\mathrm{bA}$ & 16 & eB & $12 \mathrm{gC}$ & 83 & $\mathrm{bA}$ & 32 & $\mathrm{eB}$ & 23 & $\mathrm{gC}$ \\
\hline BRS 9180 IPRO & 42 & bA & 01 & $\mathrm{gC}$ & $28 \mathrm{~dB}$ & 83 & $\mathrm{bA}$ & 03 & $\mathrm{hC}$ & 62 & $\mathrm{cB}$ \\
\hline BRASBT13-0621 & 41 & bA & 04 & $\mathrm{gC}$ & $25 \mathrm{~dB}$ & 82 & bA & 07 & $\mathrm{hC}$ & 57 & $\mathrm{cB}$ \\
\hline BRASBT13-0834 & 40 & $\mathrm{bA}$ & 03 & $\mathrm{gC}$ & $08 \mathrm{gB}$ & 80 & $\mathrm{bA}$ & 06 & $\mathrm{hC}$ & 18 & $g B$ \\
\hline BRASBT13-0016 & 39 & $\mathrm{bA}$ & 24 & $\mathrm{cB}$ & $04 \mathrm{hC}$ & 77 & $c A$ & 50 & $\mathrm{cB}$ & 13 & $\mathrm{hC}$ \\
\hline BRASBT13-0528 & 39 & bA & 16 & eB & $20 \mathrm{eB}$ & 79 & $\mathrm{bA}$ & 33 & eB & 41 & $\mathrm{eB}$ \\
\hline BRASBT13-0646 & 39 & bA & 17 & eB & $04 \mathrm{hC}$ & 81 & $\mathrm{bA}$ & 33 & $\mathrm{eB}$ & 09 & $\mathrm{hC}$ \\
\hline BRASBT13-0872 & 38 & $\mathrm{bA}$ & 04 & $g B$ & $05 \mathrm{hB}$ & 76 & $\mathrm{cA}$ & 07 & $\mathrm{hB}$ & 16 & $\mathrm{hB}$ \\
\hline BRS 9383 IPRO & 37 & bA & 25 & $\mathrm{cB}$ & $37 \mathrm{bA}$ & 75 & $\mathrm{cA}$ & 57 & $\mathrm{bB}$ & 78 & $\mathrm{bA}$ \\
\hline BRASBT13-0691 & 34 & $\mathrm{cA}$ & 15 & eB & $04 \mathrm{hC}$ & 74 & $c A$ & 29 & $\mathrm{eB}$ & 12 & $\mathrm{hC}$ \\
\hline Sambaiba 05 IPRO & 32 & $\mathrm{cB}$ & 12 & $\mathrm{fC}$ & 41 aA & 65 & $\mathrm{~dB}$ & 29 & $\mathrm{eC}$ & 87 & $\mathrm{aA}$ \\
\hline BRRY45-16300 & 32 & $\mathrm{cA}$ & 14 & $\mathrm{eC}$ & $25 \mathrm{~dB}$ & 66 & $\mathrm{dA}$ & 33 & $\mathrm{eC}$ & 51 & $\mathrm{dA}$ \\
\hline BRASBT13-0758 & 32 & $c A$ & 13 & $\mathrm{fB}$ & 01 iC & 65 & $\mathrm{dA}$ & 27 & $\mathrm{fB}$ & 06 & $\mathrm{iC}$ \\
\hline BRASBT13-0225 & 32 & $c A$ & 33 & $\mathrm{aA}$ & $02 \mathrm{iB}$ & 64 & $\mathrm{dA}$ & 69 & $\mathrm{aA}$ & 05 & $i B$ \\
\hline MSoy 7739 IPRO & 28 & $d A$ & 05 & $\mathrm{gC}$ & $14 \mathrm{fB}$ & 58 & $\mathrm{dA}$ & 14 & $g C$ & 28 & $f B$ \\
\hline BRS 9280 & 27 & $\mathrm{dA}$ & 10 & $\mathrm{fC}$ & $15 \mathrm{fB}$ & 54 & $\mathrm{dA}$ & 21 & $\mathrm{fC}$ & 31 & $f B$ \\
\hline BRASRR12-10657 & 27 & $\mathrm{dA}$ & 15 & $\mathrm{eC}$ & $23 \mathrm{eB}$ & 55 & $\mathrm{dA}$ & 32 & $\mathrm{eB}$ & 49 & $\mathrm{dA}$ \\
\hline BRRY45-16301 & 26 & $d B$ & 07 & $g C$ & 40 aA & 53 & $\mathrm{~dB}$ & 14 & $\mathrm{gC}$ & 84 & $\mathrm{aA}$ \\
\hline BRASBT13-0641 & 26 & $\mathrm{dA}$ & 14 & eB & $05 \mathrm{hC}$ & 53 & $\mathrm{dA}$ & 29 & $\mathrm{eB}$ & 09 & $\mathrm{hC}$ \\
\hline BRASRR12-14296 & 24 & eA & 24 & $c A$ & $09 \mathrm{gB}$ & 50 & $\mathrm{~dB}$ & 62 & $\mathrm{bA}$ & 21 & $\mathrm{gC}$ \\
\hline BRRY45-16283 & 24 & $\mathrm{eB}$ & 02 & $\mathrm{gC}$ & 43 aA & 48 & $\mathrm{~dB}$ & 03 & $\mathrm{hC}$ & 89 & $\mathrm{aA}$ \\
\hline BRASBT13-0698 & 24 & eA & 20 & $\mathrm{dAB}$ & $16 \mathrm{fB}$ & 48 & $\mathrm{dA}$ & 40 & $d A B$ & 34 & $f B$ \\
\hline BRASBT13-0553 & 23 & eA & 11 & $f B$ & 01 iC & 49 & $\mathrm{dA}$ & 23 & $f B$ & 02 & $\mathrm{iC}$ \\
\hline BRASRR12-13666 & 22 & eA & 04 & $g C$ & $11 \mathrm{gB}$ & 44 & $\mathrm{fA}$ & 07 & $\mathrm{hC}$ & 25 & $g B$ \\
\hline BRASRR12-13039 & 21 & eA & 04 & $\mathrm{gB}$ & $25 \mathrm{dA}$ & 43 & $f B$ & 09 & $\mathrm{hC}$ & 54 & $\mathrm{dA}$ \\
\hline BRASBT13-0176 & 21 & eA & 13 & $\mathrm{fB}$ & $05 \mathrm{hC}$ & 42 & fA & 26 & $\mathrm{fB}$ & 13 & $\mathrm{hC}$ \\
\hline BRASBT13-0590 & 20 & fA & 03 & $g B$ & 01 iB & 41 & $f A$ & 05 & $\mathrm{hB}$ & 03 & $\mathrm{iB}$ \\
\hline BRASBT13-0715 & 19 & $f B$ & 28 & bA & $05 \mathrm{hC}$ & 37 & & 61 & $\mathrm{bA}$ & 11 & $\mathrm{hC}$ \\
\hline BRS 7680 & 18 & $\mathrm{fA}$ & 03 & $g B$ & 01 iB & 41 & $\mathrm{fA}$ & 08 & $\mathrm{hB}$ & 03 & $\mathrm{iB}$ \\
\hline BRASBT13-0200 & 14 & $\mathrm{gC}$ & 20 & $\mathrm{~dB}$ & $32 \mathrm{cA}$ & 29 & $\mathrm{gC}$ & 43 & $\mathrm{~dB}$ & 71 & $\mathrm{bA}$ \\
\hline BRS 7380 & 09 & $g A$ & 03 & $g B$ & 02 iB & 19 & $g A$ & 16 & $\mathrm{gB}$ & 05 & $\mathrm{iC}$ \\
\hline CV\% & & 07 & & 3,07 & 13,07 & & 86 & 12, & & 12, & \\
\hline
\end{tabular}


"Médias seguidas por letras distintas, minúsculas na coluna e maiúsculas na linha, diferem entre si pelos testes de Scott-Knott e Tukey a $5 \%$ de probabilidade.

Marcos Filho (2015) observou que a avaliação de vigor e germinação tem a finalidade de monitorar o potencial fisiológico das sementes, principalmente para controle de empresas produtoras de sementes de soja uma vez que o limite para comercialização estabelecido no Brasil é de $80 \%$ ou mais de germinação do lote.

Marcos Filho (2013) também observou que quando o desvio de condições ambientais for muito alto, menor será a ligação entre os testes de vigor e os de emergência de plântulas em campo, pois existem vários fatores que podem influenciar na qualidade fisiológica das sementes, como por exemplo, os genótipos utilizados, as condições climáticas de cada local, dentre outros. Com isso, só será possível verificar a qualidade fisiológica das sementes, após a emergência das plântulas no campo.

$\mathrm{O}$ ambiente FSP se destacou por obter valores de primeira contagem de 41, 43 e $40 \%$ de vigor e germinação de 87,89 e $84 \%$ respectivamente para os genótipos Sambaiba 05 IPRO, BRRY45-16283 e BRRY45-16301 em relação às demais. O genótipo BRASBT13-0762 se destacou por apresentar germinação de $81 \%$, porém, não teve destaque na primeira contagem de germinação por apresentar $34 \%$ de vigor, se diferenciando estatisticamente das anteriores (Tabela $3)$.

Vinhal-Freitas et al. (2011) observaram que embora o teste de germinação seja padronizado, não funciona como aspecto de qualidade fisiológica, embora a semente germine, isso não a define como uma semente vigorosa. Assim como no caso dos genótipos MSoy 8372 IPRO e BRASBT13-0762 que apresentaram alta germinação e baixo vigor em relação aos demais genótipos dos ambientes.

Assim como as demais características analisadas, o teste de emergência em campo e o índice de velocidade de emergência expressos na Tabela 4 apresentaram diferença significativa entre os genótipos avaliados e os diferentes ambientes.

Podendo se destacar o genótipo MSoy 8372 IPRO que apresentou 95\% de emergência em campo e maior velocidade de emergência no ambiente $A B$, por ser a linhagem que se destacou estatisticamente com altos valores de vigor e germinação quando comparada aos demais genótipos (Tabela 4).

Por ser uma linhagem com sementes de maior vigor, que possui emergência homogênea e rápida, pode contribuir significativamente na obtenção de plantas por ambiente satisfatório como explica Marcos Filho (2013). Caso sejam utilizadas sementes menos vigorosas, as chances de obter emergência lenta ou heterogenia como explica o autor, provoca grandes problemas no desenvolvimento da planta além de redução na produção.

TABELA 4. Médias de emergência de plântulas em campo (EC, em \%) e velocidade de emergência em campo (VE, índice) de 35 genótipos produzidas em três ambientes

\begin{tabular}{|c|c|c|c|c|c|c|}
\hline \multirow{2}{*}{ GENÓTIPOS } & \multicolumn{3}{|c|}{ EC } & \multicolumn{3}{|c|}{ VE } \\
\hline & $\mathbf{A B}$ & $\mathrm{FL}$ & FSP & $\overline{A B}$ & $\mathrm{FL}$ & FSP \\
\hline MSoy 8372 IPRO & $95 \mathrm{aA}^{*}$ & $65 \mathrm{~dB}$ & $54 \mathrm{fC}$ & 29 aA & $13 \mathrm{gB}$ & $11 \mathrm{hC}$ \\
\hline BRASBT13-0843 & $83 \mathrm{bA}$ & $61 \mathrm{eC}$ & $77 \mathrm{aB}$ & $19 \mathrm{gA}$ & $13 \mathrm{eC}$ & $15 \mathrm{aB}$ \\
\hline BRASBT13-0762 & $81 \mathrm{cA}$ & $57 \mathrm{fC}$ & $72 \mathrm{bB}$ & $21 \mathrm{cA}$ & $12 \mathrm{hC}$ & $13 \mathrm{eB}$ \\
\hline BRS 8781 & $77 \mathrm{dA}$ & $59 \mathrm{fB}$ & $23 \mathrm{kC}$ & $18 \mathrm{iA}$ & $13 \mathrm{gB}$ & $4 \mathrm{rC}$ \\
\hline BRS 9280 & $74 \mathrm{eA}$ & $2 \mathrm{vC}$ & $31 \mathrm{jB}$ & $22 \mathrm{bA}$ & $1 \mathrm{aC}$ & $6 \mathrm{oB}$ \\
\hline BRASBT13-0646 & $73 \mathrm{fA}$ & $35 \mathrm{jB}$ & $1 \mathrm{nC}$ & $20 \mathrm{eA}$ & $7 \mathrm{nB}$ & $0 \mathrm{tC}$ \\
\hline
\end{tabular}

ENCICLOPÉDIA BIOSFERA, Centro Científico Conhecer - Goiânia, v.15 n.27; p. 2392018 


\begin{tabular}{|c|c|c|c|c|c|c|c|c|c|c|c|c|}
\hline BRASBT13-0621 & 72 & fA & 21 & $\mathrm{oC}$ & 61 & $\mathrm{~dB}$ & 21 & $d A$ & 4 & $\mathrm{rC}$ & 11 & $g B$ \\
\hline Sambaiba 05 IPRO & 66 & $g A$ & 43 & $\mathrm{hC}$ & 45 & $\mathrm{hB}$ & 19 & $\mathrm{fA}$ & 10 & kB & 9 & $\mathrm{iC}$ \\
\hline BRS 9180 IPRO & 65 & $\mathrm{hA}$ & 17 & $\mathrm{qB}$ & 65 & $c A$ & 19 & $\mathrm{hA}$ & 4 & $\mathrm{sC}$ & 13 & $\mathrm{~dB}$ \\
\hline BRASBT13-0528 & 63 & $\mathrm{iA}$ & 35 & jC & 37 & jB & 15 & oA & 8 & $\mathrm{mB}$ & 7 & $\mathrm{nC}$ \\
\hline BRRY45-16283 & 62 & $\mathrm{iA}$ & 5 & $\mathrm{uC}$ & 7 & $\mathrm{mB}$ & 16 & kB & 1 & $w C$ & 1 & sB \\
\hline BRASBT13-0758 & 60 & jA & 23 & $\mathrm{nB}$ & 0 & $\mathrm{nC}$ & 17 & jA & 5 & $q B$ & 0 & $\mathrm{tC}$ \\
\hline BRASRR12-10657 & 57 & $\mathrm{kA}$ & 33 & kB & 7 & $\mathrm{mC}$ & 16 & IA & 8 & $\mathrm{mB}$ & 1 & $\mathrm{sC}$ \\
\hline BRS 9383 IPRO & 56 & $\mathrm{kC}$ & 65 & $d B$ & 76 & $a A$ & 17 & $\mathrm{jA}$ & 15 & $\mathrm{cB}$ & 14 & $\mathrm{bC}$ \\
\hline BRASBT13-0054 & 53 & IB & 73 & $\mathrm{aA}$ & 53 & $f B$ & 14 & $\mathrm{pB}$ & 17 & $\mathrm{aA}$ & 9 & jC \\
\hline BRASBT13-0016 & 52 & IA & 53 & $g A$ & 0 & $\mathrm{nB}$ & 16 & $\mathrm{~mA}$ & 12 & iB & 0 & $\mathrm{tC}$ \\
\hline BRASBT13-0872 & 51 & $\mathrm{~mA}$ & 17 & $\mathrm{qB}$ & 0 & $\mathrm{nC}$ & 13 & rA & 3 & $\mathrm{uB}$ & 0 & $\mathrm{tC}$ \\
\hline BRRY45-16300 & 50 & $\mathrm{mB}$ & 15 & rC & 77 & $\mathrm{aA}$ & 15 & $\mathrm{nA}$ & 3 & $\mathrm{vC}$ & 13 & $\mathrm{cB}$ \\
\hline BRASRR12-13666 & 49 & $\mathrm{nB}$ & 71 & bA & 45 & $\mathrm{hC}$ & 13 & $q B$ & 14 & $c A$ & 9 & $\mathrm{kC}$ \\
\hline BRASBT13-0691 & 49 & $\mathrm{nA}$ & 37 & iB & 1 & $\mathrm{nC}$ & 11 & $\mathrm{tA}$ & 9 & IB & 0 & $\mathrm{tC}$ \\
\hline BRRY45-16301 & 44 & oB & 17 & $\mathrm{qC}$ & 62 & $\mathrm{dA}$ & 12 & sA & 4 & $\mathrm{tB}$ & 12 & $f A$ \\
\hline BRASBT13-0834 & 43 & $\mathrm{pA}$ & 23 & $n B$ & 0 & $\mathrm{nC}$ & 10 & $\mathrm{uA}$ & 5 & $q B$ & 0 & $\mathrm{tC}$ \\
\hline BRASBT13-0225 & 41 & $q B$ & 67 & $c A$ & 1 & $\mathrm{nC}$ & 8 & wB & 15 & bA & 0 & $\mathrm{tC}$ \\
\hline BRASBT13-0698 & 41 & $q C$ & 59 & $\mathrm{fA}$ & 45 & $\mathrm{hB}$ & 9 & zB & 13 & $\mathrm{fA}$ & 9 & IC \\
\hline BRASRR12-14296 & 39 & rB & 61 & eA & 19 & IC & 10 & vB & 14 & $d A$ & 4 & $q C$ \\
\hline BRASBT13-0176 & 39 & rA & 25 & $\mathrm{mB}$ & 0 & $\mathrm{nC}$ & 10 & $x A$ & 6 & $\mathrm{pB}$ & 0 & $t B$ \\
\hline MSoy 7739 IPRO & 37 & $\mathrm{sB}$ & 19 & $\mathrm{pC}$ & 47 & $g A$ & 10 & $x A$ & 4 & $\mathrm{rC}$ & 8 & $\mathrm{mB}$ \\
\hline BRASBT13-0641 & 37 & sA & 31 & IB & 0 & $\mathrm{nC}$ & 8 & yA & 6 & $\mathrm{pB}$ & 0 & $\mathrm{tC}$ \\
\hline BRASBT13-0590 & 25 & $\mathrm{tA}$ & 13 & rB & 0 & $\mathrm{nC}$ & 6 & $\alpha A$ & 2 & $x B$ & 0 & $\mathrm{tC}$ \\
\hline BRASBT13-0553 & 25 & tB & 31 & IA & 0 & $\mathrm{nC}$ & 6 & $\alpha \mathrm{B}$ & 6 & ao & 0 & $\mathrm{tC}$ \\
\hline BRASRR12-13039 & 11 & $\mathrm{uB}$ & 7 & $\mathrm{tC}$ & 23 & $\mathrm{kA}$ & 2 & ßB & 1 & $\mathrm{yC}$ & 4 & $\mathrm{pA}$ \\
\hline BRS 7380 & 0 & vB & 15 & rA & 0 & $\mathrm{nB}$ & 0 & $\gamma B$ & 3 & vA & 0 & $t B$ \\
\hline BRS 7680 & 0 & vB & 9 & sA & 0 & $\mathrm{nB}$ & 0 & $\gamma B$ & 2 & $z A$ & 0 & tB \\
\hline BRASBT13-0715 & 0 & vB & 59 & fA & 1 & $\mathrm{nB}$ & 0 & $\gamma B$ & 13 & eA & 0 & $\mathrm{tB}$ \\
\hline BRASBT13-0200 & 0 & $\mathrm{vC}$ & 53 & $\mathrm{gB}$ & 59 & $\mathrm{eA}$ & 0 & $\gamma \mathrm{C}$ & 12 & já & 11 & $\mathrm{hB}$ \\
\hline CV\% & & 94 & & 94 & & 94 & & 91 & & 91 & & 91 \\
\hline
\end{tabular}

"Médias seguidas por letras distintas, minúsculas na coluna e maiúsculas na linha, diferem entre si pelos testes de Scott-Knott e Tukey a $5 \%$ de probabilidade, respectivamente.

No ambiente FL, o percentual de emergência em campo teve destaque para o genótipo BRASBT13-0054 que apresentou 73\% e 17,39 de índice de velocidade de emergência podendo ser explicado por apresentar baixo vigor e germinação.

No ambiente FSP os melhores percentuais de emergência em campo ocorreram nos genótipos BRS 9180 IPRO, BRRY45-16300 e BRASBT13-0843 que apresentaram emergência de 76 e $77 \%$ evidenciando apenas a linhagem BRASBT13-0843 com valor superior de velocidade de emergência com 15,20, por ter maior vigor e germinação, se diferenciando estatisticamente os genótipos BRS 9180 IPRO e BRRY45-16300 por apresentarem velocidade de emergência inferior e serem sementes menos vigorosas.

Embora sejam realizados vários testes para determinação de vigor em sementes de soja, o teste de condutividade elétrica (Tabela 5), ainda se destaca como explicado por Barbosa (2012).

TABELA 5. Valores médios de condutividade elétrica (CE; CE24, em $\mu \mathrm{cm}^{-1} \mathrm{~g}^{-1}$ ), obtidos de sementes de 35 genótipos de soja produzidas em três ambientes no cerrado de Roraima

\begin{tabular}{|c|c|c|c|c|c|c|}
\hline \multirow{2}{*}{ GENÓTIPOS } & \multicolumn{3}{|c|}{ CE } & \multicolumn{3}{|c|}{ CE24 } \\
\hline & AB & FL & FSP & AB & $\mathrm{FL}$ & FSP \\
\hline & $\begin{aligned} 94,94 \mathrm{fC} \\
103,35 \mathrm{fB}\end{aligned}$ & $\begin{array}{ll}250,47 & \text { bA } \\
193,16 & \text { eA }\end{array}$ & $\begin{array}{ll}132,00 & \mathrm{iB} \\
184,48 & \mathrm{gA}\end{array}$ & $\begin{array}{ll}39,86 & \mathrm{gC} \\
41,23 & \mathrm{gC}\end{array}$ & $\begin{array}{rl}101,65 & \mathrm{cA} \\
81,22 & \mathrm{eA}\end{array}$ & $\begin{array}{ll}52,68 & \mathrm{hB} \\
72,48 & \mathrm{fB}\end{array}$ \\
\hline & $107,02 \mathrm{fC}$ & 170,71 fA & $145,67 \mathrm{hB}$ & $42,98 \mathrm{gC}$ & 70,55 fA & $57,61 \mathrm{gB}$ \\
\hline
\end{tabular}




\begin{tabular}{|c|c|c|c|c|c|c|c|c|c|c|c|}
\hline-0762 & ,43 & fA & & gA & & iA & 43,79 & gB & 57 & gA & \\
\hline & & fC & & $\mathrm{fB}$ & 187,18 & $\mathrm{gA}$ & 27 & & 62 & & 76,54 \\
\hline SBT13-0528 & 134,38 & $\mathrm{eB}$ & 104,74 & $\mathrm{hC}$ & 197,89 & $\mathrm{fA}$ &, 18 & $\mathrm{eB}$ & 56 & $\mathrm{gC}$ &, 70 \\
\hline RS 8781 & 140,24 & $\mathrm{eC}$ & 157,24 & $f B$ & 187,87 & $g A$ & 6,55 & $e B$ & 67,20 & $f A$ & \\
\hline ЗТ13-0054 & 2,33 & $\mathrm{eC}$ & 197,97 & eA & 165,10 & $\mathrm{hB}$ & & & & eA & \\
\hline T13-0758 & 2,34 & $\mathrm{eB}$ & 145,86 & gB & 199,98 & $f A$ & 8,22 & $e B$ & 61,86 & $g B$ & \\
\hline ЗТ13-0646 & 151,36 & $\mathrm{~dB}$ & 138,98 & gB & 219,83 & eA & 9,58 & $e B$ & & $\mathrm{gB}$ & \\
\hline & 2,73 & $\mathrm{~dB}$ & 174,57 & $\mathrm{fA}$ & 180, & $g A$ & 2,91 & $d B$ & & fA & \\
\hline & & $\mathrm{dC}$ & & $c A$ & & $\mathrm{eB}$ & & & & $c A$ & \\
\hline & & $\mathrm{dB}$ & & $d A$ & 162,80 & $\mathrm{hB}$ & & $d B$ & 90,02 & $d A$ & \\
\hline & & $\mathrm{dC}$ & & fA & 198,22 & $f B$ & & $\mathrm{dC}$ & & bA & \\
\hline & & $d A$ & & $d A$ & & $\mathrm{hB}$ & & $d B$ & & $d A$ & \\
\hline & & $\mathrm{dB}$ & 42 & $\mathrm{aA}$ & & $\mathrm{hB}$ & & & & $\mathrm{aA}$ & \\
\hline IPRO & 163,43 & $\mathrm{cB}$ & 218 & $d A$ & 125, & iC & & $d B$ & & $d A$ & \\
\hline & & $\mathrm{cC}$ & & eA & 182,24 & gB & & $\mathrm{cB}$ & & eA & \\
\hline & & $c A$ & & fA & & hA & & $\mathrm{dAB}$ & & fA & \\
\hline & 7,94 & $\mathrm{cC}$ & 194 & $\mathrm{eB}$ & & $d A$ & & $\mathrm{dC}$ & & $\mathrm{eB}$ & \\
\hline & & $\mathrm{cB}$ & & $d B$ & & $d A$ & & & & $d A$ & \\
\hline & & $\mathrm{cB}$ & & $\mathrm{gC}$ & & $\mathrm{eA}$ & & & & $\mathrm{gC}$ & \\
\hline & & $\mathrm{cB}$ & & $\mathrm{eB}$ & & $d A$ & & & & $\mathrm{eB}$ & \\
\hline & & $\mathrm{cB}$ & 3 & $d A$ & & hB & & & & $d A$ & \\
\hline & & $\mathrm{cB}$ & & $\mathrm{gC}$ & & $\mathrm{cA}$ & & & & $g B$ & \\
\hline & & $\mathrm{cC}$ & & bA & & $e B$ & & & & $\mathrm{cA}$ & \\
\hline & 172,76 & $c A$ & 20 & $c A$ & & $\mathrm{dA}$ & & d & & $\mathrm{cA}$ & 44 \\
\hline & 173,73 & $\mathrm{cC}$ & 208 & $d B$ & 225,65 & eA & & $\mathrm{cB}$ & & $d A$ & \\
\hline & & $c B$ & & $f B$ & & $f A$ & & & & $\mathrm{fB}$ & \\
\hline & 204,72 & $\mathrm{bB}$ & & $\mathrm{eB}$ & 274,10 & $\mathrm{bA}$ & & & & $e B$ & 104,96 \\
\hline 3666 & 213,08 & bA & 190, & $\mathrm{eB}$ & 204,76 & $\mathrm{fAE}$ & 93 & & 06 & eA & 83,96 \\
\hline & 213,63 & & 237 & $\mathrm{cA}$ & 198,38 & $f B$ & & & & $\mathrm{cA}$ & \\
\hline & & & & $\mathrm{cB}$ & & $a A$ & & & & $\mathrm{cB}$ & 123,01 \\
\hline & & & & $\mathrm{fB}$ & & $\mathrm{hC}$ & & & & $f B$ & 60,40 \\
\hline BRASRR12-13039 & 229,18 & $\mathrm{aA}$ & 201,37 & $e B$ & 191,93 & gB & 89,83 & $\mathrm{aA}$ & 81,77 & $e B$ & 79,25 \\
\hline & & & - & & 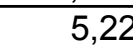 & & & & 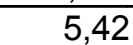 & & \\
\hline
\end{tabular}

"Médias seguidas por letras distintas, minúsculas na coluna e maiúsculas na linha, diferem entre si pelos testes de Scott-Knott e Tukey a $5 \%$ de probabilidade, respectivamente.

Os resultados da avaliação da condutividade elétrica e condutividade elétrica 24h tiveram diferença significativa como verifica-se na Tabela 5. Podem-se destacar os genótipos MSoy 8372 IPRO, BRS 9180 IPRO, BRS 9383 IPRO, BRASBT13-0762 e BRASBT13-0843 por apresentarem estatisticamente os menores valores nas leituras, indicando maior vigor em relação aos demais. Pois, revela menor desorganização dos sistemas de membranas no ambiente $A B$. Por ser um teste que avalia a integridade das membranas celulares, em decorrência da deterioração das sementes, relacionando a quantidade de íons lixiviados em soluções de embebição que indicam o alto potencial fisiológico (MARTINS et al., 2016; CASTRO et al., 2016).

No ambiente $\mathrm{FL}$, se diferenciou nos valores de condutividade o genótipo BRASBT13-0528 que apresentou 104,74 $\mu \mathrm{S} \mathrm{m}^{-1} \mathrm{~g}^{-1}$, sendo a linhagem de melhor vigor produzida. Apesar da diferença significativa no ambiente FSP, com valores variando de 125,23 a $313,85 \mu \mathrm{S} \mathrm{cm}^{-1} \mathrm{~g}^{-1}$ na condutividade e de 50,20 a $123,01 \mu \mathrm{S}$ $\mathrm{cm}^{-1} \mathrm{~g}^{-1}$ na CE24, são considerados valores altos que comprometem a qualidade fisiológica das sementes. Destacaram-se os genótipos BRS 9180 IPRO, Sambaiba 05 IPRO e BRASBT13-0762, por apresentarem os menores valores nas avaliações, se tornando os genótipos de melhor vigor do ambiente.

\section{CONCLUSÕES}


A MSoy 8372 IPRO produz sementes com maior vigor no ambiente Água Boa-AB, enquanto a BRASBT13-0225 produz sementes vigorosas na Fazenda Livramento, e a Sambaiba 05 IPRO no ambiente Fazenda São Pedro. Nenhum dos 35 genótipos apresenta maior vigor nos três ambientes. $O$ ambiente Fazenda Livramento produz mais sementes e com menor vigor.

\section{REFERÊNCIAS}

BARBOSA, R. M.; LEÃO, É. F.; CAPRIO, C. H.; VIEIRA, R. D. Teste de condutividade elétrica em sementes de maracujazeiro-amarelo. Revista Brasileira de Fruticultura, v. 34, n. 2, p. 646-651, 2012. Disponivel em: <http://dx.doi.org/10.1590/S0100-29452012000200043> Doi: S010029452012000200043

BRASIL. Ministério da Agricultura, Pecuária e Abastecimento. Regras para análise de sementes. Brasília: MAPA: ACS, 2009. 399 p.

CASTRO, E. M.; OLIVEIRA, J. A.; LIMA, A. E.; SANTOS, H. O.; BARBOSA, J. I. L.. Physiological quality of soybean seeds produced under artificial rain in the pre-harvesting period. Journal of Seed Science, v. 38, n. 1, p. 14-21, 2016. Disponivel em: <hhttp://dx.doi.org/10.1590/2317-1545v38n1154236> Doi: 10.1590/2317-1545v38n1154236

COMPANHIA NACIONAL DE ABASTECIMENTO - CONAB. 2017. Disponível em: <http://www.conab.gov.br/conabweb/download/soja/produçao.pdf>. Acesso em: 01 de dez. 2017.

FRANÇA-NETO, J. D. B., KRZYZANOWSKI, F. C., HENNING, A. A., de PÁDUA, G. P., Lorini, I., \& HENNING, F. A.. Tecnologia da produção de semente de soja de alta qualidade. Londrina: Embrapa Soja, 2016. 82 p.

MAGUIRE, J. D. Speed of germination-aid in selection and evaluation for seedling emergence and vigor. Crop Science, v.2, n.1, p. 176-177, 1962.

MARCOS FILHO, J. Fisiologia de sementes de plantas cultivadas. Londrina: ABRATES, 2. ed., 2015, 660p.

MARCOS FILHO, J. Importância do potencial fisiológico da semente de soja. Informativo Abrates, v. 23, n. 1, p. 21-23, 2013.

MARTINS, C. C.; UNÊDA-TREVISOLI, S. H.; MÔRO, G. V.; VIEIRA, R. D. Metodologia para seleção de linhagens de soja visando germinação, vigor e emergência em campo. Revista Ciência Agronômica, v. 47, n. 3, p. 455-461, 2016. Disponivel em: <http://dx.doi.org/10.5935/1806-6690.2016005> DOI: 10.5935/18066690.20160055 
SILVA, T. A.; SILVA, P. B.; SILVA, E. A. A.; NAKAGAWA, J. Y.; CAVARIANI, C. Condicionamento fisiológico de sementes de soja, componentes de produção e produtividade. Ciência Rural, v. 46, n. 2, p. 227-232, 2016.Disponivel em: <http://dx.doi.org/10.1590/0103-8478cr20141736> Doi: $10.1590 / 0103-8478 c r 20141736$

SMIDERLE, O. J.; GIANLUPPI, D.; GIANLUPPI, V.; SOUZA, A. G. Variabilidade em componentes da produção de soja (Glycine max (L.) Merrill.) BRS 8381 em diferentes níveis de calagens e densidade de plantas no cerrado de Roraima, Brasil. Revista Colombiana de Investigaciones Agroindustriales, v. 3, p. 49-55, 2016. Disponivel em: <https://doi.org/10.23850/issn.2422-0582> Doi: 10.23850/issn.2422-0582

SMIDERLE, O. J.; SOUZA, A. G.; PEDROSO, C. Â.; SILVA, T. J.; SOUZA, A. A. Correlação entre massa e vigor de sementes de Pochota fendleri armazenadas em diferentes espaçamentos. Revista de Ciências Agrárias, v. 41, n. 1, p. 93-99, 2018. Disponivel em: <http://dx.doi.org/10.19084/RCA17221 > Doi: 10.19084/RCA17221

VINHAL-FREITAS, I. C.; JUNIOR, J. E. G.; SEGUNDO, J. P.; VILARINHO, M. S.. Germinação e vigor de sementes de soja classificadas em diferentes tamanhos. Agropecuária Técnica, v. 32, n. 1, p. 108-114, 2011. Disponivel em: < https://doi.org/10.25066/agrotec.v32i1.9567>. Doi: 10.25066/agrotec.v32i1.9567 